\title{
A Stability Result for Interconnections of Nonlinear Systems with "Mixed" Small Gain and Passivity Properties
}

\author{
Wynita M. Griggs, Brian D. O. Anderson, Alexander Lanzon and Michael C. Rotkowitz
}

\begin{abstract}
A feedback interconnection consisting of two nonlinear systems is shown to be input-output stable when a "mixed" small gain and passivity assumption is placed on each of the systems. The "mixed" small gain and passivity property captures the well-known notions of passivity and small gain associated with systems: the property can be appropriately reduced to an input and output strictly passive system description; or alternatively can be reduced to a description of a system with small, finite gain. More importantly, the property captures a concept of "blending" of the small gain and passivity ideas. This concept of "blending" can be visualized, for example, by considering linear time-invariant systems that exhibit passivetype properties at, say low frequencies; and lose these passivetype properties but have small gain at high frequencies.
\end{abstract}

\section{INTRODUCTION}

A desired property of a feedback interconnection of two nonlinear systems is that the interconnection is input-output stable [1]. To determine stability, one typically places assumptions on the two nonlinear systems in the interconnection; and then shows that, if the closed-loop system's inputs belong to some class of functions, then the errors and outputs also belong to the same class of functions [2]. To illustrate, a negative feedback interconnection is shown in Fig. 1, where $M_{1}$ and $M_{2}$ are operators acting on the errors $e_{1}$ and $e_{2}$, respectively, to produce outputs $y_{1}$ and $y_{2}$, respectively.

The small gain and passivity theorems are two of the most important results in the input-output stability theory of interconnected systems. The small gain theorem states that if

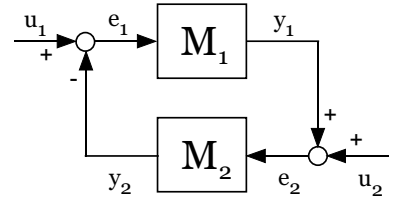

Fig. 1. Interconnection of $M_{1}$ and $M_{2}$.

This work was supported by ARC Discovery-Projects Grants (DP0342683, DP0664427) and National ICT Australia. National ICT Australia is funded through the Australian Government's Backing Australia's Ability initiative, in part through the Australian Research Council.

W. Griggs, B. Anderson and M. Rotkowitz are with Department of Information Engineering, Research School of Information Sciences and Engineering, The Australian National University, Canberra ACT 0200, Australia. B. Anderson is also with National ICT Australia Limited, Locked Bag 8001, Canberra ACT 2601, Australia.

A. Lanzon is with Control Systems Group, School of Electrical and Electronic Engineering, The University of Manchester, Sackville Street Building, Manchester M60 1QD, UK.

Corresponding author: Wynita M. Griggs, Department of Information Engineering, Research School of Information Sciences and Engineering, The Australian National University, Canberra ACT 0200, Australia wynita.griggs@anu.edu.au the product of the gains of two stable systems, interconnected as shown in Fig. 1, is less than one, then the feedback interconnection of the two systems is stable [2]-[5]. The passivity theorem guarantees stability of a feedback interconnection of two stable systems if, for instance, both of the systems are passive, and one of them is input strictly passive with finite gain [2]-[4], [6]. However, there exist many situations where stability of an interconnection cannot be determined by use of the small gain or passivity theorems because the assumptions made in the theorems are not appropriately matched to the systems in the feedback interconnection in question.

Consider the negative feedback interconnection of two single-input, single-output (SISO), linear time-invariant (LTI) systems with transfer functions $m_{1}(s)=\frac{3}{(s+1)(s+2)}$ and $m_{2}(s)=\frac{13}{(s+3)(s+4)}$. The Nyquist diagrams of these transfer functions are shown in Fig's. 2 and 3, respectively. Obviously, $m_{1}(s)$ and $m_{2}(s)$ are not in a form that allows

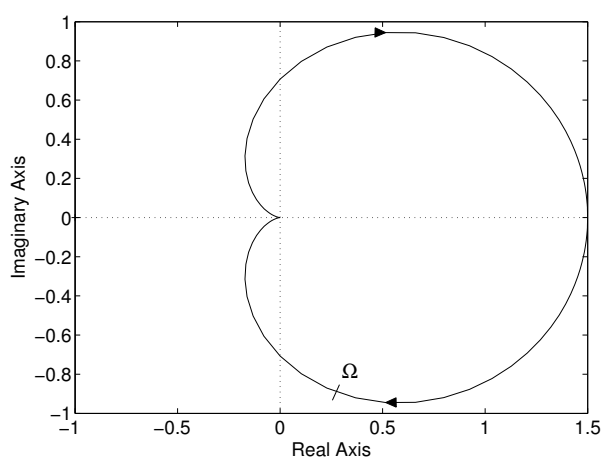

Fig. 2. Nyquist diagram of $m_{1}(s)$.

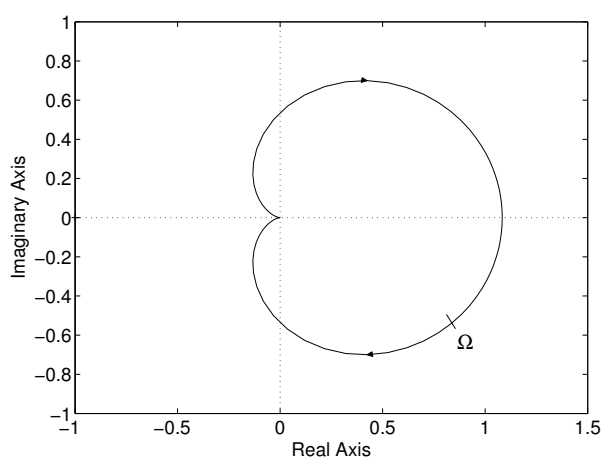

Fig. 3. Nyquist diagram of $m_{2}(s)$. 
treatment of closed-loop stability by the small gain or passivity theorems. Of course, stability of an interconnection of two LTI systems can be determined using one of the many alternative techniques available. For instance, one needs only to check that the transfer function matrix mapping input signals to error signals is input-output stable (provided that the feedback-loop is well-posed). Alternatively, one can check that the Nyquist diagram of the cascade of the two systems does not encircle the point $-1+j 0$. Note in the example provided that, since in some frequency range $[0, \Omega]$ the systems are "passive", and in the frequency range $[\Omega, \infty)$ the systems have "gain less than one", (a property which we will refer to as the "mixed" small gain and passivity property), there is no way that the Nyquist diagram of the cascade can encircle $-1+j 0$. Accordingly, the feedback interconnection of the two systems is stable. So while stability of LTI system interconnections is often easily determined, this example provides insight into an interesting class of (possibly nonlinear) systems, exhibiting what we will call a "mixed" small gain and passivity property, for which we would like to obtain stability results.

Obtaining stability results regarding such a class of systems has practical applicability. For example, it has been observed that high frequency dynamics can frequently destroy the passivity property of an otherwise passive system. A celebrated controversy in adaptive control [7] depended on the observation that passivity conditions normally forming part of the hypotheses of the proofs of convergence of certain adaptive control algorithms should not be assumed to be valid in practice (because high frequency dynamics often neglected for modelling purposes will always be present in a real system). Failure of the passivity condition invalidated the applicability of the associated theorem on the algorithm convergence to most real-life applications, and left a cloud hanging over the real-life use of the algorithm. Simulations of [7] confirmed that adverse behavior could occur when high frequency dynamics were explicitly taken into account.

The book [8] (see also [9] and [10]) described tools for establishing stability of adaptive systems of the type examined in [7]; that is, where "passivity" properties hold only for low frequency signals. Stability is established if additionally, and in a rough manner of speaking, "gains" are small at high frequencies, ie: a small gain property in the sense of the small gain theorem holds in the frequency band where the passivity condition fails. In other words, there is an important class of applications in which passivity and small gain ideas have to be blended.

A result ensuring stability of an interconnection of two multi-input, multi-output (MIMO), LTI systems, where each system exhibits a "mixed" small gain and passivity frequency domain property, was reported in [11] and [12]. We now seek to derive a stability result for interconnected, causal, nonlinear systems. As mentioned above, such a result would have practical applicability; it would also be an interesting extension to the nonlinear stability tools of passivity and small gain.

The paper is divided into the following sections. In Sec- tion II, the feedback interconnection under consideration is described in detail. In Section III, the concept of a "mixed" small gain and passivity property for nonlinear systems is defined. The closed-loop stability result is stated in Section IV and is the main contribution of this paper. Conclusions and intended future developments are provided in Section V.

\section{Notation}

The field of real numbers is denoted by $\mathbb{R}$. Suppose that $\mathcal{X}$ and $\mathcal{Y}$ are real inner product spaces. The inner product of $\mathcal{X}$ is denoted by $\langle\cdot, \cdot\rangle: \mathcal{X} \times \mathcal{X} \rightarrow \mathbb{R}$. A norm for each element of $\mathcal{X}$ is defined to be $\|f\|_{\mathcal{X}}^{2}=\langle f, f\rangle$. An important property of inner product spaces is the so-called CauchySchwarz inequality; that is $|\langle f, g\rangle| \leq\|f\|_{\mathcal{X}}\|g\|_{\mathcal{X}} \forall f, g \in \mathcal{X}$. Suppose that $\mathcal{H}$ and $\mathcal{K}$ are Hilbert spaces. For a bounded linear operator $H: \mathcal{H} \rightarrow \mathcal{K}$, the Hilbert adjoint $H^{\sim}: \mathcal{K} \rightarrow$ $\mathcal{H}$ of $H$ is defined by $\langle H h, k\rangle=\left\langle h, H^{\sim} k\right\rangle$ for all $h \in \mathcal{H}$ and $k \in \mathcal{K}$.

Let $\mathcal{L}_{2}[0, \infty)$ denote the Lebesgue space with inner product defined as

$$
\langle f, g\rangle=\int_{0}^{\infty} g^{\prime}(t) f(t) d t
$$

where the superscript $(\cdot)^{\prime}$ denotes the vector transpose. The norm of functions in $\mathcal{L}_{2}[0, \infty)$ is denoted by $\|\cdot\|$. For $T \in$ $[0, \infty)$, let $P_{T}$ denote the truncation operator. That is, for a function $f(t), 0 \leq t<\infty$,

$$
\left(P_{T} f\right)(t):=\left\{\begin{array}{ll}
f(t), & t \leq T \\
0, & t>T
\end{array} .\right.
$$

For convenience, the notation $f_{T}:=P_{T} f$ is used. We define $\langle f, g\rangle_{T}:=\left\langle f_{T}, g_{T}\right\rangle$ and note that $\left\langle f_{T}, g_{T}\right\rangle=\left\langle f_{T}, g\right\rangle=$ $\left\langle f, g_{T}\right\rangle$. Let $\mathcal{L}_{2 e}$ denote the extension of the space $\mathcal{L}_{2}[0, \infty)$, defined by $\mathcal{L}_{2 e}:=\left\{f: f_{T} \in \mathcal{L}_{2}[0, \infty) \forall T \in[0, \infty)\right\}$. Recall that the space $\mathcal{L}_{2}[0, \infty)$ satisfies the following properties:

i) The space $\mathcal{L}_{2}[0, \infty)$ is such that if $f \in \mathcal{L}_{2}[0, \infty)$, then $f_{T} \in \mathcal{L}_{2}[0, \infty) \forall T \in[0, \infty)$; and moreover, the space $\mathcal{L}_{2}[0, \infty)$ is such that $f=\lim _{T \rightarrow \infty} f_{T}$. Equivalently, the space $\mathcal{L}_{2}[0, \infty)$ is closed under the family of projections $\left\{P_{T}\right\}$.

ii) If $f \in \mathcal{L}_{2}[0, \infty)$ and $T \in[0, \infty)$, then $\left\|f_{T}\right\| \leq\|f\|$. Moreover, $\left\|f_{T}\right\|$ is a nondecreasing function of $T \in$ $[0, \infty)$.

iii) If $f \in \mathcal{L}_{2 e}$, then $f \in \mathcal{L}_{2}[0, \infty)$ if and only if $\lim _{T \rightarrow \infty}\left\|f_{T}\right\|<\infty$.

Throughout the paper, the term system will be used to refer to a mapping from $\mathcal{L}_{2 e}$ into $\mathcal{L}_{2 e}$, which satisfies a causality condition. An operator $M: \mathcal{L}_{2 e} \rightarrow \mathcal{L}_{2 e}$ is causal if $P_{T} M P_{T}=P_{T} M$ for all $T \in[0, \infty)$. An operator $M$ : $\mathcal{L}_{2 e} \rightarrow \mathcal{L}_{2 e}$ is anticausal if $\left(I-P_{T}\right) M\left(I-P_{T}\right)=\left(I-P_{T}\right) M$ for all $T \in[0, \infty)$. A system mapping $\mathcal{L}_{2 e}$ into $\mathcal{L}_{2 e}$ is inputoutput $\mathcal{L}_{2}$-stable if the output belongs to $\mathcal{L}_{2}[0, \infty)$ whenever the input belongs to $\mathcal{L}_{2}[0, \infty)$ [3]. For simplicity, inputoutput $\mathcal{L}_{2}$-stability will be referred to as input-output stability or stability when the context is clear. It is assumed that all systems considered are relaxed systems (that is, they have zero initial state). 
The operator $I: \mathcal{X} \rightarrow \mathcal{X}$, defined by $I x:=x$ for all $x \in$ $\mathcal{X}$, denotes the identity operator. The operator $0: \mathcal{X} \rightarrow \mathcal{Y}$, defined by $0 x:=0$ for all $x \in \mathcal{X}$ (where 0 denotes the zero vector from $\mathcal{Y}$ ), denotes the zero operator. The following acronyms are used throughout the paper: LTI - linear timeinvariant; SISO - single-input, single-output; MIMO - multiinput, multi-output; RHP - right half plane; RHS - right-hand side; LHS - left-hand side.

\section{FEEDBACK SYSTEM DESCRIPTION}

We wish to derive an input-output stability result for the feedback interconnection shown in Fig. 1. This feedback interconnection is described by the equations

$$
\begin{array}{ll}
e_{1}=u_{1}-y_{2} & y_{1}=M_{1} e_{1} \\
e_{2}=u_{2}+y_{1} & y_{2}=M_{2} e_{2}
\end{array}
$$

where $u_{1}, u_{2} \in \mathcal{L}_{2 e}$ are the (external) input signals; $e_{1}, e_{2} \in$ $\mathcal{L}_{2 e}$ are the error signals; and $y_{1}, y_{2} \in \mathcal{L}_{2 e}$ are the output signals. The operators $M_{1}$ and $M_{2}$ are assumed to causally map $\mathcal{L}_{2 e}$ into $\mathcal{L}_{2 e}$. Furthermore, $M_{1}$ and $M_{2}$ each have associated with them a "mixed" small gain and passivity property (defined formally in Section III).

This property captures concepts of passivity or small gain normally associated with a system: the general description of the property given in Section III can be reduced to describe a (possibly input and) output strictly passive system; alternatively, the general description of the property given in Section III can be reduced to describe a system with small gain (of less than or equal to one). What is interesting is that the property additionally captures a concept of "blending" of the small gain and passivity ideas. This concept of "blending" is best visualized if we focus for a moment on SISO, LTI systems. Nyquist plots of SISO, LTI systems with "mixed" small gain and passivity properties lie in the region consisting of the union of the RHP and the inside of the unit circle, as shown in Fig. 4. The "mixed" small gain and passivity frequency domain property for SISO, LTI systems was formally defined in [11] and [12] as follows: there exist constants $0 \leq \epsilon<1, k>0$ and $l>0$ such that

$$
\begin{aligned}
-\langle\hat{m} \hat{f},(k \alpha+1-\alpha) \hat{m} \hat{f}\rangle & +2\langle\hat{m} \hat{f}, \alpha \hat{f}\rangle \\
& -\langle\hat{f},(l \alpha-\epsilon(1-\alpha)) \hat{f}\rangle \geq 0
\end{aligned}
$$

$\forall \hat{f} \in \mathcal{H}_{2}{ }^{1}$; where $\hat{m} \in \mathcal{R H}_{\infty}{ }^{2}$ and $\alpha(\omega)$ is a real, continuous, even function of frequency that is: i) equal to

${ }^{1} \mathcal{H}_{2}$ denotes the real frequency domain Hardy space in which

$$
\|\hat{f}\|=\left\{\frac{1}{2 \pi} \int_{-\infty}^{\infty} \hat{f}^{*}(j \omega) \hat{f}(j \omega) d \omega\right\}^{\frac{1}{2}}
$$

and the superscript $(\cdot)^{*}$ denotes the complex conjugate transpose. $\mathcal{H}_{2}$ is a Hilbert space under the inner product

$$
\langle\hat{f}, \hat{g}\rangle=\frac{1}{2 \pi} \int_{-\infty}^{\infty} \hat{g}^{*}(j \omega) \hat{f}(j \omega) d \omega .
$$

\footnotetext{
${ }^{2} \mathcal{L}_{\infty}$ is a Banach space of matrix- (or scalar-) valued functions that are essentially bounded on $j \mathbb{R}$. The Hardy space, $\mathcal{H}_{\infty}$, is the closed subspace of $\mathcal{L}_{\infty}$ with functions that are analytic and bounded in the open RHP. $\mathcal{R} \mathcal{H}_{\infty}$ denotes the subspace of $\mathcal{H}_{\infty}$ whose transfer functions are proper and real rational, and consequently, are analytic and bounded in the closed RHP.
}

one on frequency intervals for which the system described by $\hat{m}(s)$ is "input and output strictly passive"; ii) equal to zero on frequency intervals for which the system described by $\hat{m}(s)$ has "gain less than one"; and iii) is strictly greater than zero and strictly less than one on frequency intervals for which the system described by $\hat{m}(s)$ is "input and output strictly passive with gain less than one". When the feedback interconnection of two such SISO, LTI systems is considered, the "mixed" small gain and passivity stability result further requires that there exist common frequency intervals over which both systems are "passive" (and may or may not have "small gain"); and that the remaining frequency intervals are intervals over which both systems have "small gain" (and may or may not be "passive"). (Refer to [11] and [12] for further details.) Examples of two systems that satisfy these requirements were given in Section I.

So we intend for the "mixed" small gain and passivity property to capture a class of causal, nonlinear systems that is larger than the class of (possibly input and) output strictly passive systems together with the class of systems with small gain. Strictness and non-strictness of the "mixed" small gain and passivity property will be dealt with formally in later sections. Similarly to the passivity and small gain theorems, one of the systems in the feedback interconnection is required to have a strict form of the "mixed" small gain and passivity property associated with it.

Well-posedness of the feedback interconnection corresponds to the existence and uniqueness of solutions $e_{1}, e_{2}$ and $y_{1}, y_{2}$ for each choice of $u_{1}, u_{2}$ (and one may also wish to include requirements of causality and continuous dependence) [13]. References [1], [14] describe conditions to impose on the operators $M_{1}$ and $M_{2}$ to guarantee wellposedness of the feedback-loop. We do not discuss wellposedness further in this paper; we assume that the feedback interconnection illustrated in Fig. 1 has a unique solution.

\section{THE “MIXED” SMALL GAIN AND PASSIVITY PROPERTY}

As mentioned above, the "mixed" small gain and passivity property can be thought of as a "blending" of the concepts of passivity and small gain. We first recall the concepts of finite gain and passivity.

Definition 1: [3] A system $M: \mathcal{L}_{2 e} \rightarrow \mathcal{L}_{2 e}$ is said to have a finite gain if there exist constants $\bar{\epsilon} \geq 0$ and $\eta \geq 0$,

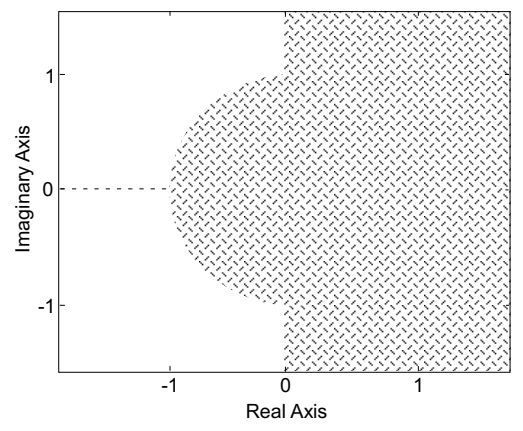

Fig. 4. Union of RHP and inner unit circle. 
such that

$$
\left\|(M f)_{T}\right\| \leq \bar{\epsilon}\left\|f_{T}\right\|+\eta
$$

for all input signals $f \in \mathcal{L}_{2 e}$ and all $T \in[0, \infty)$.

The constant $\eta$ is called the bias term and is included to allow for the case where $M f \neq 0$ when $f=0$ [3]. Clearly, if there do exist constants $\bar{\epsilon}$ and $\eta$ such that (1) holds, then $\bar{\epsilon}$ is not uniquely defined. We call the gain of $M$ the number $\epsilon$ defined by

$$
\epsilon=\inf \left\{\bar{\epsilon} \in \mathbb{R}_{+}: \exists \eta \text { such that inequality (1) holds }\right\}
$$

(see [2, Section III.2]). Systems with finite gain are said to be finite-gain stable [3]. Obviously, if a system has finite gain, then the system is input-output stable.

Definition 2: [3] A system $M: \mathcal{L}_{2 e} \rightarrow \mathcal{L}_{2 e}$ is said to be input and output strictly passive if there exist constants $k>0, l>0$ and $m \in \mathbb{R}$ such that

$$
\langle M f, f\rangle_{T} \geq k\left\|(M f)_{T}\right\|^{2}+l\left\|f_{T}\right\|^{2}+m
$$

for all input signals $f \in \mathcal{L}_{2 e}$ and all $T \in[0, \infty)$. The system $M$ is said to be input strictly passive if it satisfies (2) with $k=0$; output strictly passive if it satisfies (2) with $l=0$; and, passive if it satisfies (2) with $k=l=0$.

The bias term $m$ is included to account for the possible effect of energy stored in the system at $t=0$ [3]. Note that input and output strict passivity is equivalent to input strict passivity with finite gain [6], [15], [16]. We now define (the strict version of) a "mixed" small gain and passivity property.

Definition 3: Let $\Gamma: \mathcal{L}_{2}[0, \infty) \rightarrow \mathcal{L}_{2}[0, \infty)$ and $\mathrm{B}$ : $\mathcal{L}_{2}[0, \infty) \rightarrow \mathcal{L}_{2}[0, \infty)$ be causal, bounded, linear operators such that

$$
\Gamma^{\sim} \Gamma+\mathrm{B}^{\sim} \mathrm{B}=I .
$$

(Note that a causal operator mapping $\mathcal{L}_{2}[0, \infty)$ into $\mathcal{L}_{2}[0, \infty)$ immediately extends to map $\mathcal{L}_{2 e}$ into $\mathcal{L}_{2 e}$.) Then a system $M: \mathcal{L}_{2 e} \rightarrow \mathcal{L}_{2 e}$ is said to have a strict "mixed" small gain and passivity property if there exist constants $0 \leq \epsilon<1$, $k>-1, l \geq 0$ and $\zeta \geq 0$ such that

$$
\begin{array}{r}
-\langle M f, M f\rangle_{T}+\epsilon\langle\Gamma f, \Gamma f\rangle_{T}-k\langle\mathrm{~B} M f, \mathrm{~B} M f\rangle_{T} \\
+2\langle\mathrm{~B} M f, \mathrm{~B} f\rangle_{T}-l\langle\mathrm{~B} f, \mathrm{~B} f\rangle_{T}+\zeta \geq 0
\end{array}
$$

for all input signals $f \in \mathcal{L}_{2 e}$ and all $T \in[0, \infty)$.

The "mixed" small gain and passivity property captures the concepts of passivity or small gain normally associated with a system as follows. If $\Gamma=\mathbf{0}$, then (4) describes a (possibly input and) output strictly passive system. If $\mathrm{B}=\mathbf{0}$, then (4) describes a system with gain less than one. The description of the "mixed" small gain and passivity property additionally captures a concept of "blending" of the small gain and passivity ideas. In the case of LTI systems for example, if $\Gamma$ is close to 0 at low frequencies and close to 1 at high frequencies (at least in magnitude), then the mixed property in qualitative terms corresponds to the system being passive at low frequencies and having small gain at high frequencies. References [11] and [12] extensively illustrate the concept of "blending" of the small gain and passivity ideas in the frequency domain.
A consequence of a system having a strict "mixed" small gain and passivity property is that the system is guaranteed to have finite gain.

Lemma 4: A system $M: \mathcal{L}_{2 e} \rightarrow \mathcal{L}_{2 e}$ with a strict "mixed" small gain and passivity property associated with it (in the sense of Definition 3) has finite gain.

Proof: Let $\Gamma_{T}:=P_{T} \Gamma$ and $\mathrm{B}_{T}:=P_{T} \mathrm{~B}$, and note that

$$
\Gamma_{T}^{\sim} \Gamma_{T}+\mathrm{B}_{T}^{\sim} \mathrm{B}_{T} \leq I
$$

$\forall T \in[0, \infty)$. (That is, $\left\langle x,\left(\Gamma_{T}^{\sim} \Gamma_{T}+\mathrm{B}_{T}^{\sim} \mathrm{B}_{T}\right) x\right\rangle \leq\langle x, I x\rangle$ for all $x \in \mathcal{L}_{2}[0, \infty)$ and $T \in[0, \infty)$.) Due to the causality of $\Gamma$ and $\mathrm{B}$, and the linearity and anticausality of $\Gamma^{\sim}$ and $\mathrm{B}^{\sim}$, inequality (4) can be rewritten as

$$
\begin{aligned}
& \text { LHS : }=\left\|(M f)_{T}\right\|^{2}+k\left\langle M f, \mathrm{~B}_{T}^{\sim} \mathrm{B}_{T} M f\right\rangle_{T}-\zeta \\
& \leq \epsilon\left\langle f, \Gamma_{T}^{\sim} \Gamma_{T} f\right\rangle_{T}-l\left\langle f, \mathrm{~B}_{T}^{\sim} \mathbf{B}_{T} f\right\rangle_{T}+2\left\langle M f, \mathrm{~B}_{T}^{\sim} \mathrm{B}_{T} f\right\rangle_{T} \\
& \leq \epsilon\left\langle f, \Gamma_{T}^{\sim} \Gamma_{T} f\right\rangle_{T}+l\left\langle f, \mathrm{~B}_{T}^{\sim} \mathbf{B}_{T} f\right\rangle_{T}+2\left\langle M f, \mathrm{~B}_{T}^{\sim} \mathbf{B}_{T} f\right\rangle_{T} \\
& =\text { : RHS. }
\end{aligned}
$$

Let $\psi=\max \{\epsilon, l\}$. The RHS of the preceding inequality is less than or equal to

$$
\begin{aligned}
& \psi\left\langle f,\left(\Gamma_{T}^{\sim} \Gamma_{T}+\mathbf{B}_{T}^{\sim} \mathbf{B}_{T}\right) f\right\rangle_{T}+2\left\langle M f, \mathbf{B}_{T}^{\sim} \mathbf{B}_{T} f\right\rangle_{T} \\
& \leq \psi\left\|f_{T}\right\|^{2}+2\left\langle M f, \mathbf{B}_{T}^{\sim} \mathbf{B}_{T} f\right\rangle_{T} \text { (using (5)) } \\
& =\psi\left\|f_{T}\right\|^{2}+2\left\langle M f, \mathbf{B}_{T}^{\sim} \mathbf{B}_{T} f_{T}\right\rangle_{T} \text { (since } \mathbf{B} \text { is causal) } \\
& \leq \psi\left\|f_{T}\right\|^{2}+2\left\|(M f)_{T}\right\|\left\|\mathbf{B}_{T}^{\sim} \mathbf{B}_{T}\right\|\left\|f_{T}\right\| \text { (using the }
\end{aligned}
$$

Cauchy-Schwarz and submultiplicative inequalities)

$$
\leq \psi\left\|f_{T}\right\|^{2}+2\left\|(M f)_{T}\right\|\left\|f_{T}\right\|\left(\text { since }\left\|\mathrm{B}_{T}^{\sim} \mathrm{B}_{T}\right\| \leq 1\right) .
$$

The LHS of the aforementioned inequality is greater than or equal to $\phi\left\|(M f)_{T}\right\|^{2}-\zeta$, where $0<\phi \leq 1$ is some constant, as follows. If $k \geq 0$, then the LHS is greater than or equal to $\left\|(M f)_{T}\right\|^{2}-\zeta$. If $-1<k<0$, then the LHS is equal to

$$
\begin{aligned}
& \left\|(M f)_{T}\right\|^{2}-|k|\left\langle M f, \mathrm{~B}_{T}^{\sim} \mathbf{B}_{T} M f\right\rangle_{T}-\zeta \\
& =\left\|(M f)_{T}\right\|^{2}-|k|\left\langle M f, \mathbf{B}_{T}^{\sim} \mathbf{B}_{T}(M f)_{T}\right\rangle_{T}-\zeta \text { (since B is } \\
& \quad \text { causal) } \\
& \geq\left\|(M f)_{T}\right\|^{2}-|k|\left\|\mathbf{B}_{T}^{\sim} \mathbf{B}_{T}\right\|\left\|(M f)_{T}\right\|^{2}-\zeta \text { (using the }
\end{aligned}
$$

Cauchy-Schwarz and submultiplicative inequalities)

$$
\begin{aligned}
& \geq\left\|(M f)_{T}\right\|^{2}-|k|\left\|(M f)_{T}\right\|^{2}-\zeta\left(\text { since }\left\|\mathbf{B}_{T}^{\sim} \mathbf{B}_{T}\right\| \leq 1\right) \\
& =(1-|k|)\left\|(M f)_{T}\right\|^{2}-\zeta .
\end{aligned}
$$

Then $\phi:=\left\{\begin{array}{ll}1, & k \geq 0 \\ 1-|k|, & -1<k<0\end{array}\right.$ and we have

$$
\phi\left\|(M f)_{T}\right\|^{2} \leq \psi\left\|f_{T}\right\|^{2}+2\left\|(M f)_{T}\right\|\left\|f_{T}\right\|+\zeta .
$$

Since $\phi>0$, we can write this in the form

$$
\left\|(M f)_{T}\right\|^{2} \leq 2 \bar{\phi}\left\|f_{T}\right\|\left\|(M f)_{T}\right\|+\bar{\phi}\left(\psi\left\|f_{T}\right\|^{2}+\zeta\right)
$$

where $\bar{\phi}:=\frac{1}{\phi}$, and so

$$
\begin{aligned}
\left\|(M f)_{T}\right\| & \leq \bar{\phi}\left\|f_{T}\right\|+\sqrt{\bar{\phi}^{2}\left\|f_{T}\right\|^{2}+\bar{\phi}\left(\psi\left\|f_{T}\right\|^{2}+\zeta\right)} \\
& \leq \bar{\phi}\left\|f_{T}\right\|+\sqrt{\bar{\phi}^{2}\left\|f_{T}\right\|^{2}+\bar{\phi} \psi\left\|f_{T}\right\|^{2}}+\sqrt{\bar{\phi} \zeta}
\end{aligned}
$$




$$
=\left(\bar{\phi}+\sqrt{\bar{\phi}^{2}+\bar{\phi} \psi}\right)\left\|f_{T}\right\|+\sqrt{\bar{\phi} \zeta} .
$$

\section{STABILITY RESULT}

We now show that if two causal, nonlinear systems $M_{1}$ and $M_{2}$ each have associated with them a "mixed" small gain and passivity property, and furthermore, if the "mixed" small gain and passivity property associated with system $M_{2}$ is strict, then the feedback interconnection illustrated in Fig. 1 is guaranteed to be input-output stable. ${ }^{3}$ This result is the main contribution of the paper.

Theorem 5: Consider a feedback interconnection as shown in Fig. 1 and described by the equations

$$
\begin{aligned}
& e_{1}=u_{1}-M_{2} e_{2} \\
& e_{2}=u_{2}+M_{1} e_{1}
\end{aligned}
$$

where $M_{1}$ and $M_{2}$ causally map $\mathcal{L}_{2 e}$ into $\mathcal{L}_{2 e}$. Assume that for any $u_{1}$ and $u_{2}$ in $\mathcal{L}_{2}[0, \infty)$, there are solutions $e_{1}$ and $e_{2}$ in $\mathcal{L}_{2 e}$. Suppose that there exist constants $\epsilon_{1}, k_{1}, l_{1}, \zeta_{1}, \epsilon_{2}, k_{2}, l_{2}$ and $\zeta_{2}$ such that

$$
\begin{aligned}
& -\left\langle M_{1} f, M_{1} f\right\rangle_{T}+\epsilon_{1}\langle\Gamma f, \Gamma f\rangle_{T}-k_{1}\left\langle\mathrm{~B} M_{1} f, \mathrm{~B} M_{1} f\right\rangle_{T} \\
& +2\left\langle\mathrm{~B} M_{1} f, \mathrm{~B} f\right\rangle_{T}-l_{1}\langle\mathrm{~B} f, \mathrm{~B} f\rangle_{T}+\zeta_{1} \geq 0 \\
& -\left\langle M_{2} f, M_{2} f\right\rangle_{T}+\epsilon_{2}\langle\Gamma f, \Gamma f\rangle_{T}-k_{2}\left\langle\mathrm{~B} M_{2} f, \mathrm{~B} M_{2} f\right\rangle_{T} \\
& +2\left\langle\mathrm{~B} M_{2} f, \mathrm{~B} f\right\rangle_{T}-l_{2}\langle\mathrm{~B} f, \mathrm{~B} f\rangle_{T}+\zeta_{2} \geq 0
\end{aligned}
$$

$\forall f \in \mathcal{L}_{2 e}, \forall T \in[0, \infty)$, where $\Gamma$ and $\mathrm{B}$ are defined in Definition 3. Under these conditions, if

$$
\begin{array}{cc}
0 \leq \epsilon_{1} \leq 1 & l_{1}+k_{2} \geq 0 \\
0 \leq \epsilon_{2}<1 & l_{2}+k_{1} \geq 0 \\
k_{2}>-1, & l_{2} \geq 0
\end{array}
$$

then $u_{1}, u_{2} \in \mathcal{L}_{2}[0, \infty)$ imply that $e_{1}, e_{2}, M_{1} e_{1}, M_{2} e_{2} \in$ $\mathcal{L}_{2}[0, \infty)$.

To avoid confusion, note that $\Gamma$ is a single operator that appears in (8) and (9). Similarly, B is a single operator that appears in (8) and (9). ${ }^{4}$ Also note that with appropriate choices of $\Gamma$ and $\mathrm{B}$, Theorem 5 reduces to versions of the passivity theorem $(\Gamma=0)$; or the small gain theorem $(B=0)$.

The input and output signal space for the feedback interconnection shown in Fig. 1 is the product space $\mathcal{L}_{2 e} \times \mathcal{L}_{2 e}$, and the elements of the input and output signal space are $u:=\left(\begin{array}{l}u_{1} \\ u_{2}\end{array}\right)$ and $y:=\left(\begin{array}{l}y_{1} \\ y_{2}\end{array}\right)$, respectively. Inner products in these spaces are derived by summing inner products in the component spaces. The proof to Theorem 5 thus proceeds

\footnotetext{
${ }^{3}$ In fact (corresponding to the choice of constants $k_{1}$ and $l_{1}$ below), the result states that $M_{1}$ need not have a "mixed" small gain and passivity property associated with it at all, provided that the "lack" of the property is compensated by the "strength" of the "mixed" small gain and passivity property associated with $M_{2}$. The constants defined in Theorem 5 and the conditions associated with them quantify these ideas of "lack", "strength" and compensation.

${ }^{4}$ In the LTI case, this relates to a requirement that a common frequency interval can be found on which both systems in the feedback interconnection are "input and output strictly passive and have gain less than one" (see [12, Section II] for details).
}

with a summing of the inner products in (8) and (9) to derive an inner product inequality describing the feedback interconnection. Then appropriate manipulations to the inner product inequality give the desired stability result.

Proof: Truncating (6) and (7) gives

$$
\begin{aligned}
& e_{1 T}=u_{1 T}-\left(M_{2} e_{2}\right)_{T} \\
& e_{2 T}=u_{2 T}+\left(M_{1} e_{1}\right)_{T} .
\end{aligned}
$$

For any $u_{1}, u_{2} \in \mathcal{L}_{2}[0, \infty)$, for any $T \in[0, \infty)$,

$$
\begin{aligned}
& -\left\langle M_{1} e_{1}, M_{1} e_{1}\right\rangle_{T}+\epsilon_{1}\left\langle\Gamma e_{1}, \Gamma e_{1}\right\rangle_{T}-l_{1}\left\langle\mathrm{~B} e_{1}, \mathrm{~B} e_{1}\right\rangle_{T} \\
& +2\left\langle\mathrm{~B} M_{1} e_{1}, \mathrm{~B} e_{1}\right\rangle_{T}-k_{1}\left\langle\mathrm{~B} M_{1} e_{1}, \mathrm{~B} M_{1} e_{1}\right\rangle_{T}+\zeta_{1} \\
& -\left\langle M_{2} e_{2}, M_{2} e_{2}\right\rangle_{T}+\epsilon_{2}\left\langle\Gamma e_{2}, \Gamma e_{2}\right\rangle_{T}-l_{2}\left\langle\mathrm{~B} e_{2}, \mathrm{~B} e_{2}\right\rangle_{T} \\
& +2\left\langle\mathrm{~B} M_{2} e_{2}, \mathrm{~B} e_{2}\right\rangle_{T}-k_{2}\left\langle\mathrm{~B} M_{2} e_{2}, \mathrm{~B} M_{2} e_{2}\right\rangle_{T}+\zeta_{2} \\
= & -\left\langle e_{1},\left(I-\epsilon_{1} \Gamma_{T}^{\sim} \Gamma_{T}\right) e_{1}\right\rangle_{T}-\left(l_{1}+k_{2}\right)\left\langle e_{1}, \mathrm{~B}_{T}^{\sim} \mathbf{B}_{T} e_{1}\right\rangle_{T} \\
& -\left\langle e_{2},\left(I-\epsilon_{2} \Gamma_{T}^{\sim} \Gamma_{T}\right) e_{2}\right\rangle_{T}-\left(l_{2}+k_{1}\right)\left\langle e_{2}, \mathrm{~B}_{T}^{\sim} \mathbf{B}_{T} e_{2}\right\rangle_{T} \\
& -\left\langle u_{1},\left(I+k_{2} \mathrm{~B}_{T}^{\sim} \mathbf{B}_{T}\right) u_{1}\right\rangle_{T}-\left\langle u_{2},\left(I+k_{1} \mathbf{B}_{T}^{\sim} \mathbf{B}_{T}\right) u_{2}\right\rangle_{T} \\
& +2\left\langle e_{1},\left(I+k_{2} \mathbf{B}_{T}^{\sim} \mathrm{B}_{T}\right) u_{1}\right\rangle_{T}+2\left\langle e_{2},\left(I+k_{1} \mathbf{B}_{T}^{\sim} \mathbf{B}_{T}\right) u_{2}\right\rangle_{T} \\
& -2\left\langle e_{1}, \mathrm{~B}_{T}^{\sim} \mathrm{B}_{T} u_{2}\right\rangle_{T}+2\left\langle e_{2}, \mathrm{~B}_{T}^{\sim} \mathbf{B}_{T} u_{1}\right\rangle_{T}+\zeta_{1}+\zeta_{2}
\end{aligned}
$$

using (10) and (11) to substitute in for $\left(M_{2} e_{2}\right)_{T}$ and $\left(M_{1} e_{1}\right)_{T}$, respectively; and appealing to the causality of $\Gamma$ and $\mathrm{B}$, and the linearity and anticausality of $\Gamma^{\sim}$ and $\mathrm{B}^{\sim}$ while rearranging. Using (8) and (9), the LHS and thus the RHS of this equality is greater than or equal to zero, giving a new inequality. In this inequality, set $\bar{\zeta}:=\zeta_{1}+\zeta_{2}$ for convenience. Then, for any $u_{1}, u_{2} \in \mathcal{L}_{2}[0, \infty)$ and any $T \in[0, \infty)$, we know that there exist constants $\epsilon_{1}, k_{1}, l_{1}, \epsilon_{2}, k_{2}, l_{2}$ and $\bar{\zeta}$ such that

$$
\begin{aligned}
& \left\|e_{1 T}\right\|^{2}-\epsilon_{1}\left\langle e_{1}, \Gamma_{T}^{\sim} \Gamma_{T} e_{1}\right\rangle_{T}+\left(l_{1}+k_{2}\right)\left\langle e_{1}, \mathbf{B}_{T}^{\sim} \mathbf{B}_{T} e_{1}\right\rangle_{T}+ \\
& \left\|e_{2 T}\right\|^{2}-\epsilon_{2}\left\langle e_{2}, \Gamma_{T}^{\sim} \Gamma_{T} e_{2}\right\rangle_{T}+\left(l_{2}+k_{1}\right)\left\langle e_{2}, \mathbf{B}_{T}^{\sim} \mathbf{B}_{T} e_{2}\right\rangle_{T} \\
& \leq 2\left\langle e_{1}, u_{1}\right\rangle_{T}-2\left\langle e_{1}, \mathrm{~B}_{T}^{\sim} \mathrm{B}_{T} u_{2}\right\rangle_{T}+2 k_{2}\left\langle e_{1}, \mathrm{~B}_{T}^{\sim} \mathrm{B}_{T} u_{1}\right\rangle_{T}+ \\
& 2\left\langle e_{2}, u_{2}\right\rangle_{T}+2\left\langle e_{2}, \mathbf{B}_{T}^{\sim} \mathbf{B}_{T} u_{1}\right\rangle_{T}+2 k_{1}\left\langle e_{2}, \mathbf{B}_{T}^{\sim} \mathbf{B}_{T} u_{2}\right\rangle_{T}- \\
& k_{2}\left\langle u_{1}, \mathbf{B}_{T}^{\sim} \mathbf{B}_{T} u_{1}\right\rangle_{T}-\left\|u_{1 T}\right\|^{2}-k_{1}\left\langle u_{2}, \mathbf{B}_{T}^{\sim} \mathbf{B}_{T} u_{2}\right\rangle_{T}- \\
& \left\|u_{2 T}\right\|^{2}+\bar{\zeta} \text {. }
\end{aligned}
$$

The LHS of (12) is greater than or equal to

$$
\begin{aligned}
& \left\|e_{1 T}\right\|^{2}-\epsilon_{1}\left\langle e_{1}, \Gamma_{T}^{\sim} \Gamma_{T} e_{1}\right\rangle_{T}+\left\|e_{2 T}\right\|^{2}-\epsilon_{2}\left\langle e_{2}, \Gamma_{T}^{\sim} \Gamma_{T} e_{2}\right\rangle_{T} \\
& \text { (since } \left.l_{1}+k_{2} \geq 0 \text { and } l_{2}+k_{1} \geq 0\right) \\
& =\left\|e_{1 T}\right\|^{2}-\epsilon_{1}\left\langle e_{1}, \Gamma_{T}^{\sim} \Gamma_{T} e_{1 T}\right\rangle_{T}-\epsilon_{2}\left\langle e_{2}, \Gamma_{T}^{\sim} \Gamma_{T} e_{2 T}\right\rangle_{T}+ \\
& \quad\left\|e_{2 T}\right\|^{2} \text { (since } \Gamma \text { is causal) } \\
& \geq\left\|e_{1 T}\right\|^{2}-\epsilon_{1}\left\|\Gamma_{T}^{\sim} \Gamma_{T}\right\|\left\|e_{1 T}\right\|^{2}-\epsilon_{2}\left\|\Gamma_{T}^{\sim} \Gamma_{T}\right\|\left\|e_{2 T}\right\|^{2}+ \\
& \quad\left\|e_{2 T}\right\|^{2} \text { (using the Cauchy-Schwarz and } \\
& \quad \text { submultiplicative inequalities) } \\
& \geq\left\|e_{1 T}\right\|^{2}-\epsilon_{1}\left\|e_{1 T}\right\|^{2}+\left\|e_{2 T}\right\|^{2}-\epsilon_{2}\left\|e_{2 T}\right\|^{2} \text { (since } \\
& \left.\quad\left\|\Gamma_{T}^{\sim} \Gamma_{T}\right\| \leq 1\right) \\
& =\left(1-\epsilon_{1}\right)\left\|e_{1 T}\right\|^{2}+\left(1-\epsilon_{2}\right)\left\|e_{2 T}\right\|^{2} \\
& \left.\geq\left(1-\epsilon_{2}\right)\left\|e_{2 T}\right\|^{2} \text { (since } 1-\epsilon_{1} \geq 0\right) .
\end{aligned}
$$


Then

$$
\begin{aligned}
& \left(1-\epsilon_{2}\right)\left\|e_{2 T}\right\|^{2} \\
\leq & 2\left\langle e_{1}, u_{1}\right\rangle_{T}-2\left\langle e_{1}, \mathbf{B}_{T}^{\sim} \mathbf{B}_{T} u_{2}\right\rangle_{T}+2 k_{2}\left\langle e_{1}, \mathbf{B}_{T}^{\sim} \mathbf{B}_{T} u_{1}\right\rangle_{T}+ \\
& 2\left\langle e_{2}, u_{2}\right\rangle_{T}+2\left\langle e_{2}, \mathbf{B}_{T}^{\sim} \mathbf{B}_{T} u_{1}\right\rangle_{T}+2 k_{1}\left\langle e_{2}, \mathbf{B}_{T}^{\sim} \mathbf{B}_{T} u_{2}\right\rangle_{T}+ \\
& \bar{\zeta}-k_{2}\left\langle u_{1}, \mathbf{B}_{T}^{\sim} \mathbf{B}_{T} u_{1}\right\rangle_{T}-\left\|u_{1 T}\right\|^{2}-k_{1}\left\langle u_{2}, \mathbf{B}_{T}^{\sim} \mathbf{B}_{T} u_{2}\right\rangle_{T} \\
& -\left\|u_{2 T}\right\|^{2} \\
\leq & 2\left\langle e_{1}, u_{1}\right\rangle_{T}-2\left\langle e_{1}, \mathbf{B}_{T}^{\sim} \mathbf{B}_{T} u_{2}\right\rangle_{T}+2 k_{2}\left\langle e_{1}, \mathbf{B}_{T}^{\sim} \mathbf{B}_{T} u_{1}\right\rangle_{T}+ \\
& 2\left\langle e_{2}, u_{2}\right\rangle_{T}+2\left\langle e_{2}, \mathbf{B}_{T}^{\sim} \mathbf{B}_{T} u_{1}\right\rangle_{T}+2 k_{1}\left\langle e_{2}, \mathbf{B}_{T}^{\sim} \mathbf{B}_{T} u_{2}\right\rangle_{T}- \\
& k_{2}\left\langle u_{1}, \mathbf{B}_{T}^{\sim} \mathbf{B}_{T} u_{1}\right\rangle_{T}-k_{1}\left\langle u_{2}, \mathbf{B}_{T}^{\sim} \mathbf{B}_{T} u_{2}\right\rangle_{T}+\bar{\zeta} \\
\leq & 2\left|\left\langle e_{1}, u_{1}\right\rangle_{T}\right|+2\left|\left\langle e_{1}, \mathbf{B}_{T}^{\sim} \mathbf{B}_{T} u_{2}\right\rangle_{T}\right|+\left|k_{2}\right|\left\langle u_{1}, \mathbf{B}_{T}^{\sim} \mathbf{B}_{T} u_{1}\right\rangle_{T} \\
& +2\left|k_{2}\right|\left|\left\langle e_{1}, \mathbf{B}_{T}^{\sim} \mathbf{B}_{T} u_{1}\right\rangle_{T}\right|+2\left|k_{1}\right|\left|\left\langle e_{2}, \mathbf{B}_{T}^{\sim} \mathbf{B}_{T} u_{2}\right\rangle_{T}\right|+\bar{\zeta}+ \\
& 2\left|\left\langle e_{2}, u_{2}\right\rangle_{T}\right|+2\left|\left\langle e_{2}, \mathbf{B}_{T}^{\sim} \mathbf{B}_{T} u_{1}\right\rangle_{T}\right|+\left|k_{1}\right|\left\langle u_{2}, \mathbf{B}_{T}^{\sim} \mathbf{B}_{T} u_{2}\right\rangle_{T} .
\end{aligned}
$$

Letting $\rho=\max \left\{\left|k_{1}\right|,\left|k_{2}\right|\right\}$, the last inequality is less than or equal to

$$
\begin{aligned}
& 2\left|\left\langle e_{1}, u_{1}\right\rangle_{T}\right|+2\left|\left\langle e_{1}, \mathbf{B}_{T}^{\sim} \mathbf{B}_{T} u_{2}\right\rangle_{T}\right|+\rho\left\langle u_{1}, \mathbf{B}_{T}^{\sim} \mathbf{B}_{T} u_{1}\right\rangle_{T}+ \\
& 2\left|\left\langle e_{2}, u_{2}\right\rangle_{T}\right|+2\left|\left\langle e_{2}, \mathbf{B}_{T}^{\sim} \mathbf{B}_{T} u_{1}\right\rangle_{T}\right|+\rho\left\langle u_{2}, \mathbf{B}_{T}^{\sim} \mathbf{B}_{T} u_{2}\right\rangle_{T}+ \\
& 2 \rho\left(\left|\left\langle e_{1}, \mathbf{B}_{T}^{\sim} \mathbf{B}_{T} u_{1}\right\rangle_{T}\right|+\left|\left\langle e_{2}, \mathbf{B}_{T}^{\sim} \mathbf{B}_{T} u_{2}\right\rangle_{T}\right|\right)+\bar{\zeta} \\
& =2\left|\left\langle e_{1}, u_{1}\right\rangle_{T}\right|+2\left|\left\langle e_{1}, \mathbf{B}_{T}^{\sim} \mathbf{B}_{T} u_{2 T}\right\rangle_{T}\right|+\rho\left\langle u_{1}, \mathbf{B}_{T}^{\sim} \mathbf{B}_{T} u_{1 T}\right\rangle_{T} \\
& \quad+2 \rho\left(\left|\left\langle e_{1}, \mathbf{B}_{T}^{\sim} \mathbf{B}_{T} u_{1 T}\right\rangle_{T}\right|+\left|\left\langle e_{2}, \mathbf{B}_{T}^{\sim} \mathbf{B}_{T} u_{2 T}\right\rangle_{T}\right|\right)+\bar{\zeta}+ \\
& \quad 2\left|\left\langle e_{2}, u_{2}\right\rangle_{T}\right|+2\left|\left\langle e_{2}, \mathbf{B}_{T}^{\sim} \mathbf{B}_{T} u_{1 T}\right\rangle_{T}\right|+\rho\left\langle u_{2}, \mathbf{B}_{T}^{\sim} \mathbf{B}_{T} u_{2 T}\right\rangle_{T}
\end{aligned}
$$

(since $\mathrm{B}$ is causal)

$$
\begin{aligned}
\leq & 2\left\|e_{1 T}\right\|\left\|u_{1 T}\right\|+2\left\|\mathbf{B}_{T}^{\sim} \mathbf{B}_{T}\right\|\left(\left\|e_{1 T}\right\|\left\|u_{2 T}\right\|+\left\|e_{2 T}\right\|\left\|u_{1 T}\right\|\right) \\
& +2\left\|e_{2 T}\right\|\left\|u_{2 T}\right\|+\rho\left\|\mathbf{B}_{T}^{\sim} \mathbf{B}_{T}\right\|\left(\left\|u_{1 T}\right\|^{2}+\left\|u_{2 T}\right\|^{2}\right)+\bar{\zeta}+ \\
& 2 \rho\left\|\mathbf{B}_{T}^{\sim} \mathbf{B}_{T}\right\|\left(\left\|e_{1 T}\right\|\left\|u_{1 T}\right\|+\left\|e_{2 T}\right\|\left\|u_{2 T}\right\|\right) \text { (using the } \\
& \text { Cauchy-Schwarz and submultiplicative inequalities) } \\
\leq & 2 \rho\left(\left\|e_{1 T}\right\|\left\|u_{1 T}\right\|+\left\|e_{2 T}\right\|\left\|u_{2 T}\right\|\right)+\rho\left(\left\|u_{1 T}\right\|^{2}+\left\|u_{2 T}\right\|^{2}\right) \\
& +2\left(\left\|e_{1 T}\right\|+\left\|e_{2 T}\right\|\right)\left(\left\|u_{1 T}\right\|+\left\|u_{2 T}\right\|\right)+\bar{\zeta}(\text { since } \\
& \left.\left\|\mathbf{B}_{T}^{\sim} \mathbf{B}_{T}\right\| \leq 1\right) .
\end{aligned}
$$

Using (10) to eliminate $e_{1 T}$, followed by the triangle inequality, and then applying Lemma 4 to $M_{2}$, gives

$$
\begin{aligned}
\left(1-\epsilon_{2}\right)\left\|e_{2 T}\right\|^{2} & \\
\leq & 2\left(\left\|u_{1 T}\right\|+\kappa\left\|e_{2 T}\right\|+\xi+\left\|e_{2 T}\right\|\right)\left(\left\|u_{1 T}\right\|+\left\|u_{2 T}\right\|\right)+ \\
& 2 \rho\left[\left(\left\|u_{1 T}\right\|+\kappa\left\|e_{2 T}\right\|+\xi\right)\left\|u_{1 T}\right\|+\left\|e_{2 T}\right\|\left\|u_{2 T}\right\|\right]+ \\
& \rho\left(\left\|u_{1 T}\right\|^{2}+\left\|u_{2 T}\right\|^{2}\right)+\bar{\zeta}
\end{aligned}
$$

where the non-negative constants $\kappa$ and $\xi$ exist due to the boundedness of $M_{2}$. Since $1-\epsilon_{2}>0$, write (13) in the form

$$
\left\|e_{2 T}\right\|^{2} \leq 2 \bar{b}(T)\left\|e_{2 T}\right\|+\bar{c}(T) .
$$

Note that $\bar{b}(T)$ and $\bar{c}(T)$ tend to finite values $\bar{b}$ and $\bar{c}$, respectively, as $T \rightarrow \infty$, since $u_{1}, u_{2} \in \mathcal{L}_{2}[0, \infty)$. From (14)

$$
\left\|e_{2 T}\right\| \leq \bar{b}(T)+\left(\bar{b}(T)^{2}+\bar{c}(T)\right)^{\frac{1}{2}}
$$

$\forall T \in[0, \infty)$, and remains bounded as $T \rightarrow \infty$. So $e_{2} \in$ $\mathcal{L}_{2}[0, \infty)$. From Lemma 4 the same holds for $M_{2} e_{2}$, ie: $M_{2} e_{2} \in \mathcal{L}_{2}[0, \infty)$. By (6) and (7) it follows that $e_{1}$ and $M_{1} e_{1} \in \mathcal{L}_{2}[0, \infty)$.

\section{CONCLUSIONS AND FUTURE WORK}

Input-output stability for a feedback interconnection of two causal nonlinear systems was proven, where each system had a "mixed" small gain and passivity property associated with it. We indicated that this "mixed" small gain and passivity assumption reduces to a passive system description, or to a description of a system with small gain, when certain operators of the property are appropriately defined. More importantly, the property describes systems that have "blended" small gain and passivity ideas attributed to them which the small gain and passivity theorems alone cannot capture. It is well-known that nonlinear stability techniques, such as integral quadratic constraint and dissipative system concepts, also capture the notions of small gain, and alternatively, passivity. In future work, we intend that the relationship between the "mixed" small gain and passivity concept described in this paper, and other nonlinear stability techniques (including Lyapunov stability notions) be explored.

\section{ACKNOWLEDGMENTS}

The authors wish to thank Professor David Hill for his helpful comments.

\section{REFERENCES}

[1] J.C. Willems, The Analysis of Feedback Systems, The M.I.T. Press, Cambridge, MA; 1971.

[2] C.A. Desoer and M.Vidyasagar, Feedback Systems: Input-Output Properties, Academic Press, New York, NY; 1975.

[3] H.J. Marquez, Nonlinear Control Systems: Analysis and Design, John Wiley \& Sons, Hoboken, NJ; 2003.

[4] M. Green and D.J.N. Limebeer, Linear Robust Control, Prentice Hall, Englewood Cliffs, NJ; 1995.

[5] K. Zhou, J.C. Doyle and K. Glover, Robust and Optimal Control, Prentice Hall, Upper Saddle River, NJ; 1996.

[6] A.R. Teel, T.T. Georgiou, L. Praly and E. Sontag, "Input-output stability", in: W.S. Levine (Ed.), The Control Handbook, CRC Press, Boca Raton, FL; 1996, pp 895-908.

[7] C.E. Rohrs, L. Valavani, M. Athans and G. Stein, Robustness of continuous-time adaptive control algorithms in the presence of unmodeled dynamics, IEEE Trans. Autom. Control, vol. 30, no. 9, 1985, pp 881-889.

[8] B.D.O. Anderson, R.R. Bitmead, C.R. Johnson, Jr., P.V. Kokotovic, R.L. Kosut, I.M.Y. Mareels, L. Praly and B.D. Riedle, Stability of Adaptive Systems: Passivity and Averaging Analysis, The M.I.T. Press, Cambridge, MA; 1986.

[9] I.M.Y. Mareels, B.D.O. Anderson, R.R. Bitmead, M. Bodson and S.S. Sastry, "Revisiting the MIT rule for adaptive control", in Second IFAC Workshop on Adaptive Systems in Control and Signal Processing, Lund, Sweden, 1986, pp 161-166.

[10] B.D.O. Anderson, Failures of adaptive control thoery and their resolution, Communications in Information and Systems, vol. 5, no. 1, 2005, pp 1-20.

[11] W.M. Griggs, B.D.O. Anderson and A. Lanzon, A "mixed" small gain and passivity theorem in the frequency domain, Systems \& Control Letters, vol. 56, 2007, pp 596-602.

[12] W.M. Griggs, B.D.O. Anderson and A. Lanzon, "A "mixed" small gain and passivity theorem for an interconnection of linear time-invariant systems", Proceedings of the European Control Conference 2007, Kos, Greece, 2007, pp 2410-2416.

[13] M. Vidyasagar, Nonlinear Systems Analysis, Prentice Hall, Englewood Cliffs, NJ; 1993.

[14] C.J. Harris and J.M.E. Valenca, The Stability of Input-Output Dynamical Systems, Academic Press, London, UK; 1983.

[15] P.J. Moylan and D.J. Hill, Stability criteria for large-scale systems, IEEE Trans. Autom. Control, vol. 23, no. 2, 1978, pp 143-149.

[16] D. Hill and P. Moylan, The stability of nonlinear dissipative systems, IEEE Trans. Autom. Control, vol. 21, no. 5, 1976, pp 708-711. 